\title{
Identification and Classification of Indoor Plants According to Light Intensity Requirements for Botanical loT Application
}

\author{
Charlotte C. Shagol, Kwang Jin Kim*, Seung Won Han, Na Ra Jeong, Hyeon Ju Kim, Young Bin Jung, and Hyung Kwon Yun \\ Urban Agriculture Research Division, National Institute of Horticultural and Herbal Science, Rural Development Administration, \\ Wanju 55365, Korea
}

\begin{abstract}
This study aimed to provide information to urban plant growers on the best light requirement for indoor plants. Chlorophyll fluorescence analysis was used to determine the photosynthetic activity of indoor plants as affected by light intensity. Determination of the light intensity requirement for each plant was done by applying the Bayesian Michaelis-Menten equation which was obtained using the Lineweaver-Burk plot. The Vmax and $\mathrm{Km}$ values were determined, where the photosynthetic activity measured as electron transport rate (ETR) was denoted as Vmax and light intensity (photosynthetically active radiation, PAR) of $\frac{1}{2}$ maximum ETR was designated as $\mathrm{Km}$. The ETR of 93 indoor plant species were determined and $\mathrm{Vmax} / 2$ and $\mathrm{Km}$ values of each plant were calculated which resulted in seven levels of light intensity. The levels are as follows: Level 1 - more than the Km value; Levels 2 to 7 were determined using the formula: maximum $\mathrm{Km}$ value of the level $=\mathrm{Km}\left(\frac{1}{2}\right)^{\mathrm{n}-1}$ where $\mathrm{n}$ refers to the level (i.e. $\mathrm{n} 2=$ level $2, \cdots 7$ ). The best is level 1 while the most sub-optimal is level 7 . The different plants have a wide range of light intensity requirements. Majority of the plants (70) had $\frac{1}{2}$ maximum ETR at 20 up to less than $100 \mu \mathrm{mol} \mathrm{m}^{-2} \mathrm{~s}^{-1} \mathrm{PAR}$, while 13 plants at 100 to $149 \mu \mathrm{mol} \mathrm{m} \mathrm{s}^{-2}$ and only 10 plants at $150 \mathrm{up}$ to $290 \mu \mathrm{mol} \mathrm{m}^{-2} \mathrm{~s}^{-1}$. The indoor plants can be classified according to light intensity requirement: low light intensity, e.g. Dracaena sanderiana 'Gold' (20.9); moderate light intensity, e.g. Cyclamen persicum (99.1); and high light intensity, Polyscias fruticosa $\left(290.2 \mu \mathrm{mol} \cdot \mathrm{m}^{-2} \cdot \mathrm{s}^{-1}\right)$. The data generated from this study can be utilized in loT to make the information on plant cultivation and environmental conditions accessible to urban growers who use smartphones.
\end{abstract}

Keywords: indoor environment, indoor greenery, Internet-of-Things, light intensity, smart home

\section{Introduction}

In the advent of smart technologies, services for smart homes become inevitable. A smart home enables the interaction between home owners or users and appliances in the home. The need for Internet of Things (IoT) services for eHomes or smart homes is expected to increase in the coming years. IoT is a technology that tends to connect all the objects in the world to the internet (Channe et al., 2015) including home devices and appliances. The introduction of smart homes has been proposed to improve the quality of life of residents when they are at home. At present, smart homes are focused on energy efficiency and

This work was carried out with the support of "Cooperative Research Program for Agriculture, Science and Technology Development (Project No. PJ0125502018)”, Rural Development Administration, Republic of Korea.

Received: September 28, 2018, Revised: October 4, 2018, Accepted: October 11, 2018

First author: Charlotte C. Shagol, E-mail: charlotte34@korea.kr, ORCID: 0000-0003-2524-1284

*Corresponding author: Kwang Jin Kim, E-mail: kwangjin@korea.kr, ORCID: 0000-0002-2549-9720

2018 by the Society for People, Plants, and Environment. This is an Open Access article distributed under the terms of the Creative Common Attribution Non-Commercial License (http://creativecommons.org/licenses/by-nc/4.0/) which permits unrestricted non-commercial use, distribution, and reproduction in any medium, provided the original work is properly cited. 
convenient living at home (Chen et al., 2017). These maybe referred to as the traditional or first-generation smart home or Smart Home 1.0 (Chen et al., 2017).

Recently, smart technology applications have also incorporated green technologies for example making smart green buildings. Few works have introduced greeneries into smart homes, thus Chen et al. (2017) proposed an upgrade to the Smart Home 1.0 to include botanicals into the home environment in consideration of the users' emotions (Smart Home 2.0). The cultivation of ornamental plants indoors does not only include aesthetic value but also other purposes such as air purification (Kim et al., 2018; Irga et al., 2018), stress amelioration (Lee et al., 2015), or promotion of creativity (Shibata and Suzuki, 2002, 2004). For smart homes that contain indoor plants, platforms or apps that will assist them in successful indoor plant cultivation will be necessary.

Whether in a traditional house or smart house, indoor plants need optimum environment to grow properly. One major environmental factor that greatly influences plant growth is light (Pennisi, 2017). Different plants require a wide range of light intensity for proper growth and development. Indoor ornamental plants are known to favor low to medium light intensities. Knowledge of the light requirements of plants would aide growers to maximize the benefits from indoor plant cultivation. Plants are usually classified according to their light intensity requirements as follows: low-light plants, medium-light plants, high-light plants, and very high-light plants (Pennisi, 2017). Suboptimal light conditions indoors not only limit growth of the plants but air purifying capacity of the plants as well if stomatal absorption of pollutants is to be depended upon (Brilli et al., 2018). One way to investigate the effect of light intensity on plant growth is through chlorophyll fluorescence analysis. Chlorophyll fluorescence analysis has become an important tool in plant biology. It is a non-invasive way to study photochemical and nonphotochemical processes within the thylakoid membranes, chloroplasts, plant tissues, and the whole plant (Rohacek et al., 2008).

To accomplish the intended purpose of aesthetic value, improved air quality, and improved psychological and mental health brought about by indoor plants in smart homes, data is the essential material for intelligence. Therefore, it is important to have a database of plant responses to crucial environmental factors affecting plant growth and development. This study aimed to identify the light intensity requirements of different interior plants and classify these light intensities into categories that may aide in IoT services for smart homes.

\section{Materials and Methods}

\section{Plants and Growth Condition}

Ninety-three species of ornamental indoor plants in $10 \mathrm{~cm}$-diameter plastic pots containing growth media mix (Sungro®, Canada). Three individual potted plants per species were maintained as replicates and these were grown in a plant growth room with the following conditions: $23 \pm 1^{\circ} \mathrm{C} ; 12 \mathrm{~h}$ light cycle; $28 \mu \mathrm{mol} \cdot \mathrm{m}^{-2} \cdot \mathrm{s}^{-1}$ light intensity (fluorescent lamps); 40-60\% relative humidity.

\section{Chlorophyll Fluorescence Analysis}

Healthy, intact, 3rd to 4th leaves from the shoot of 2 to 3 month-old potted plants were taken as samples from each plant species per replicate. Sample leaves of each plant species were dark adapted for $20 \mathrm{~min}$ before chlorophyll fluorescence analysis using Heinz Walz IMAGING-PAM fluorometer K-5 (Figure 1). The electron transport rate (ETR) was determined by recording a light curve for each plant sample in $0,5,16,26,41,61,86,116,151,191,236$, and $286 \mu \mathrm{mol}$ $\mathrm{m}^{-2} \mathrm{~s}^{-1}$ photosynthetically active radiation (PAR). 


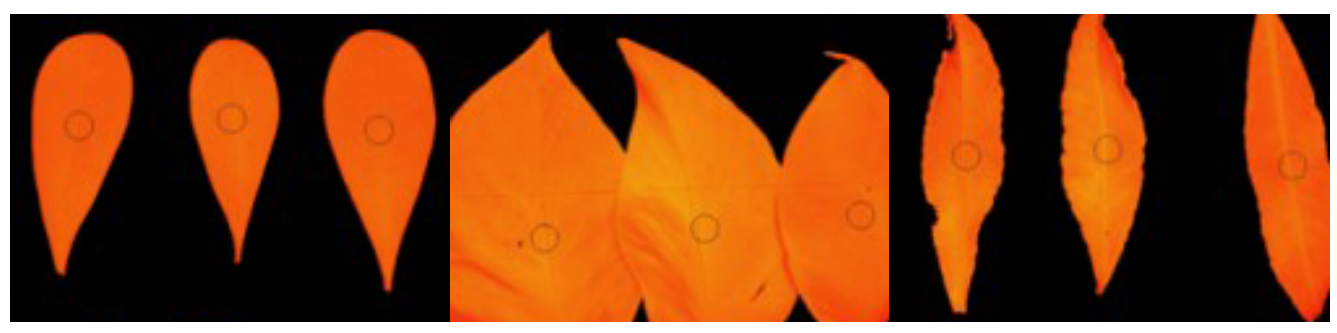

Figure 1. Chlorophyll fluorescence analysis of three leaves using pulse amplified modulation imaging. Circles on leaves are areas of interest chosen during analysis.

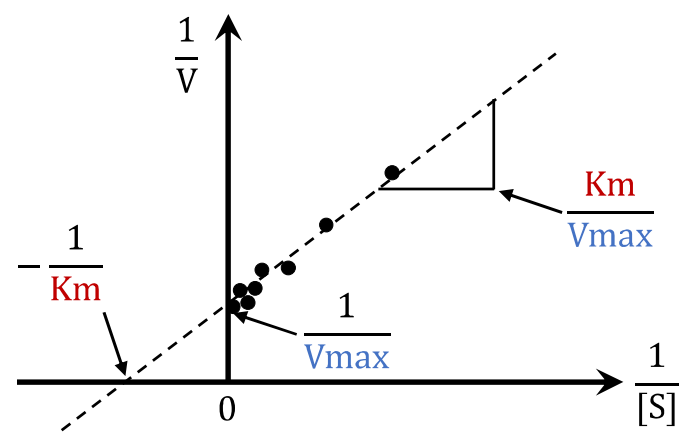

Figure 2. Lineweaver-Burk plot.

The ETR is a relative measure of the apparent electron transport rate. The ETR was computed as follows:

$$
\text { ETR }=\text { Yield } \times \text { PAR } \times 0.5 \times \text { Absorptivity }
$$

Where: absorptivity amounts to $0.84 \mu$ equivalents $\mathrm{m}^{-2} \mathrm{~s}^{-1}$

\section{Determination of Vmax and $\mathrm{Km}$}

The Michaelis-Menten equation was used to determine the relationship of ETR and different PAR (Equation 1). Vmax and $\mathrm{Km}$ values were determined by using the Lineweaver-Burk plot (Figure 2, Equation 2). In this study, ETR was designated as Vmax, and PAR as Km. Km for each plant species was determined where Vmax/2.

$$
\begin{gathered}
V_{0}=\frac{V \max [S]}{K m+[S]} \\
\frac{1}{V}=\frac{K m+[S]}{V \max [S]}=\frac{K m}{V \max } \frac{1}{[S]}+\frac{1}{V \max }
\end{gathered}
$$

Using regression equation in Microsoft Excel, the y-intercepts and slopes were determined and Vmax and Km were calculated. 


\section{Results and Discussion}

Light intensity influences various processes in the plant. Photosynthetic activity of 93 indoor plants at different PAR measured as electron transport rate (ETR) was determined by chlorophyll fluorescence analysis. ETR and PAR values were fitted in a regression equation to derive Vmax and $\mathrm{Km}$ values. A graph of determined slopes and y-intercepts of three representative plant species is shown in Figure 3.

The measured ETR and PAR where the maximum ETR is halved $(\mathrm{Km})$ of the 93 indoor plants is shown in Table 1. Among the plants measured, Dracaena sanderiana 'Gold' had the lowest ETR at the highest PAR used in the study while the highest was in Polyscias fruticosa. Several species of Dracaena registered the lowest ETR (6.5 - 7.0). However, D. sanderiana 'Virens' require almost double $\left(55.6 \mu \mathrm{mol} \cdot \mathrm{m}^{-2} \cdot \mathrm{s}^{-1}\right)$ of the $\mathrm{Km}$ value of $D$. sanderiana 'Gold' (20.9 $\left.\mu \mathrm{mol} \cdot \mathrm{m}^{-2} \cdot \mathrm{s}^{-1}\right)$. Hedera helix varieities 'Variegata', 'Harley Quinn', and 'Dream' registered similar ETR (21.0-26.0) and $\mathrm{Km}$ values $\left(82.4-85.6 \mu \mathrm{mol} \cdot \mathrm{m}^{-2} \cdot \mathrm{s}^{-1}\right)$. These species are known to require medium to high light intensities (Peninisi, 2017; Shaughnessy and Pertuit, 1999).

Plotting the ETR against a particular PAR for each plant resulted in a curve similar to the Michealis-Menten reaction curve (Figure 4). For each plant, seven levels of PAR were formed using the formula, $\mathrm{Km}(1 / 2)^{n-1}$. Level 1 is designated as the best level, where PAR is highest; while Level 7 is the most sub-optimal PAR level for the plant.

Table 2 shows the upper limit of the PAR levels for each level of each plant studied. According to Pinyuh et al. (2012), perhaps the most limiting environmental factor in indoor plant growth is lack of adequate light since as the natural light enters a home, this decrease very quickly. Categorizing the light intensity into levels may aide in data interpretation when used in IoT applications.

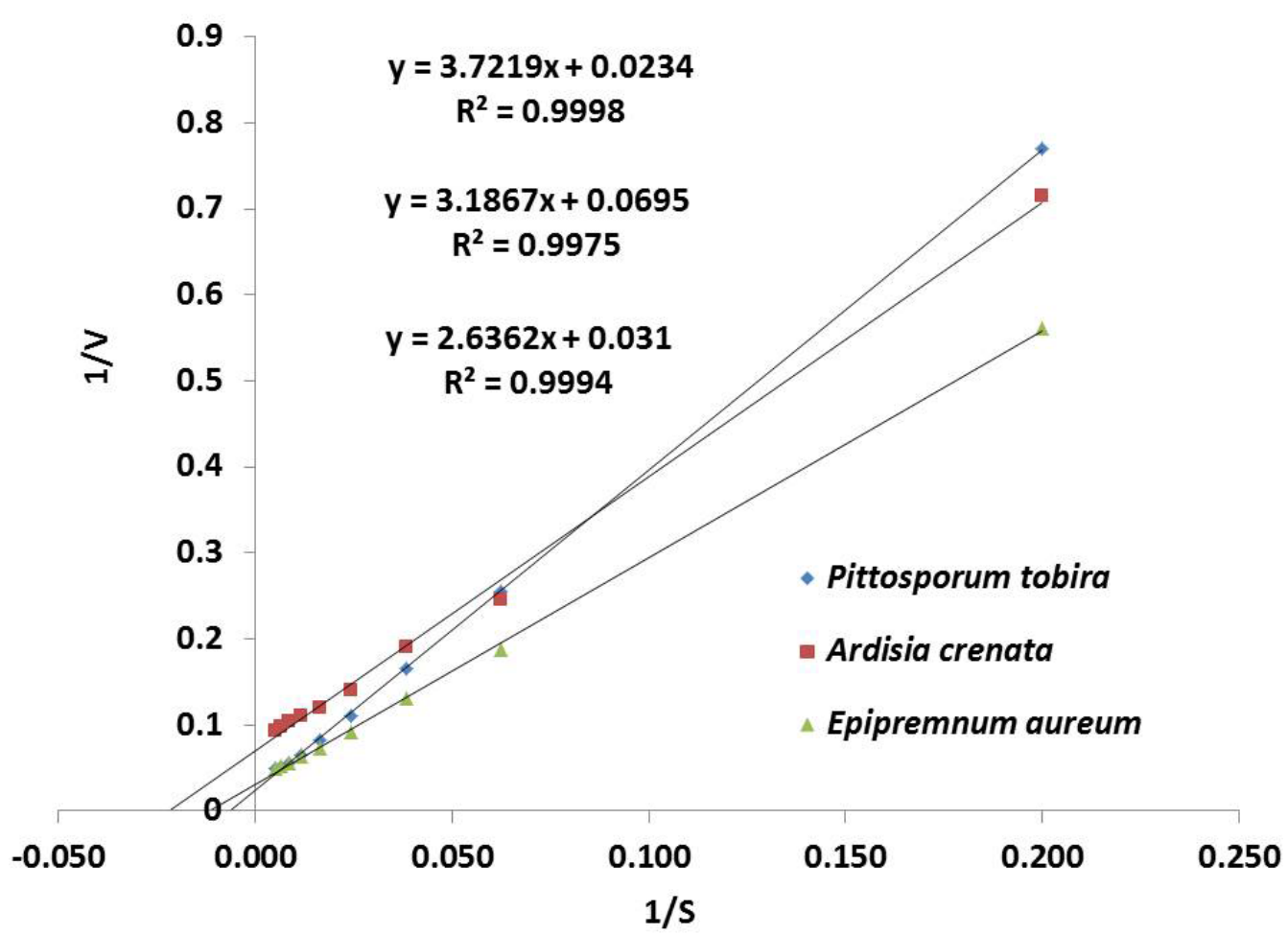

Figure 3. Lineweaver-Burk plot of three representative indoor plant species. Graph, slopes and y-intercepts were generated in Microsoft Excel. 
Table 1. Vmax and $\mathrm{Km}$ values of 93 indoor plant species

\begin{tabular}{|c|c|c|c|}
\hline Botanical name & Common name & $\begin{array}{l}\text { Vmax } \\
(\mathrm{ETR})\end{array}$ & $\begin{array}{c}\mathrm{Km} \\
\left(\mu \mathrm{mol} \cdot \mathrm{m}^{-2} \cdot \mathrm{s}^{-1}\right)\end{array}$ \\
\hline$\overline{\text { Dracaena sanderiana 'Gold' }}$ & Belgian evergreen & $6.5 \pm 0.4$ & $20.9 \pm 1.3$ \\
\hline Guzmania 'Ice Cream' & Tufted airplant & $6.9 \pm 0.5$ & $21.8 \pm 1.6$ \\
\hline Dracaena sanderiana 'Victoria' & Belgian evergreen 'Victoria' & $7.9 \pm 0.8$ & $25.2 \pm 2.7$ \\
\hline Dracaena fragrans 'Massangeana Compacta' & Mass cane & $7.0 \pm 0.6$ & $26.4 \pm 5.1$ \\
\hline Asparagus setaceus 'Nanus' & Asparagus fern & $8.8 \pm 0.6$ & $28.3 \pm 2.0$ \\
\hline Begonia rex & Painted-leaf begonia & $8.8 \pm 2.0$ & $28.4 \pm 6.7$ \\
\hline Chamaedorea elegans & Neanthe bella palm & $9.1 \pm 1.6$ & $34.6 \pm 8.2$ \\
\hline Philodendron 'Congo' & Green congo philodendron & $9.7 \pm 0.6$ & $31.1 \pm 1.9$ \\
\hline Peperomia argyreia & Watermelon peperomia & $9.8 \pm 0.6$ & $31.5 \pm 2.0$ \\
\hline Saintpaulia ionantha & African violet & $9.8 \pm 1.5$ & $31.6 \pm 4.8$ \\
\hline Syngonium podophyllum 'Pixie' & Red aglaonema & $9.9 \pm 1.9$ & $32.1 \pm 6.3$ \\
\hline Aglaonema 'Siam Aurora' & Butterfly plant & $6.7 \pm 2.1$ & $32.1 \pm 10.3$ \\
\hline Dryopteris crassirhizoma & Thick-stemmed wood fern & $10.0 \pm 0.7$ & $32.2 \pm 2.4$ \\
\hline Peperomia clusiifolia 'Isabella' & Isabella peperomia & $10.1 \pm 2.0$ & $32.5 \pm 6.5$ \\
\hline Codiaeum variegatum & Variegated croton & $10.6 \pm 1.0$ & $34.2 \pm 0.4$ \\
\hline Gaultheria procumbens L. & Eastern teaberry & $11.0 \pm 0.7$ & $35.4 \pm 2.5$ \\
\hline Fittonia verschaffelti 'Redstar' & Red star nerve plant & $11.2 \pm 2.9$ & $36.4 \pm 9.6$ \\
\hline Heteropanax fragrans & Happy tree & $11.3 \pm 0.2$ & $36.6 \pm 0.6$ \\
\hline Calathea insignis & Prayer plant & $11.8 \pm 0.3$ & $38.4 \pm 1.1$ \\
\hline Cordyline terminalis & Good luck tree & $12.0 \pm 0.7$ & $38.9 \pm 2.5$ \\
\hline Selaginella martensii & Variegated spikemoss & $12.1 \pm 0.9$ & $39.5 \pm 2.9$ \\
\hline Nertera granadensis & Coral bead plant & $12.2 \pm 2.3$ & $39.8 \pm 7.7$ \\
\hline Rhapis excelsa & Broadleaf lady palm & $13.2 \pm 2.5$ & $43.0 \pm 8.3$ \\
\hline Ficus elastica & Rubber plant & $11.6 \pm 1.0$ & $43.8 \pm 6.7$ \\
\hline Begonia semperflorens & Wax begonia & $13.6 \pm 1.0$ & $44.3 \pm 3.2$ \\
\hline Codiaeum variegatum 'Mammy' & Mammy croton & $13.6 \pm 1.0$ & $44.4 \pm 3.4$ \\
\hline Asplenium nidus 'Avis' & Bird's-nest fern & $14.1 \pm 2.7$ & $45.9 \pm 8.9$ \\
\hline Trachelospermum asiaticum Nakai & Asiatic jasmine & $14.1 \pm 1.0$ & $46.0 \pm 3.4$ \\
\hline Ficus pumila L. 'Variegata' & Variegated creeping fig & $14.4 \pm 0.5$ & $47.0 \pm 1.8$ \\
\hline Ficus lyrata & Fiddle-leaf fig & $14.6 \pm 2.4$ & $47.6 \pm 7.8$ \\
\hline Ardisia pusilla A.DC. & Small coralberry & $14.7 \pm 3.1$ & $48.1 \pm 10.2$ \\
\hline Fittonia albivenis Brummitt 'Whitestar' & White star nerve plant & $12.9 \pm 0.8$ & $49.0 \pm 8.0$ \\
\hline Aglaonema 'Redgold' & Red gold Chinese evergreen & $16.1 \pm 2.1$ & $52.8 \pm 7.1$ \\
\hline Ardisia crenata Sims & Christmas berry & $16.2 \pm 3.3$ & $52.9 \pm 11.0$ \\
\hline Schefflera arboricola 'HongKong' & Parasol plant & $16.9 \pm 1.6$ & $55.3 \pm 5.4$ \\
\hline Dracaena sanderiana 'Virens' & Lucky bamboo & $11.4 \pm 2.1$ & $55.6 \pm 10.7$ \\
\hline Coffea arabica & Coffee tree & $17.5 \pm 0.7$ & $57.3 \pm 2.4$ \\
\hline Peperomia puteolata & Parallel peperomia & $13.0 \pm 0.9$ & $57.4 \pm 10.5$ \\
\hline Pilea glauca 'Greizy' & Silver sprinkles & $17.7 \pm 2.7$ & $57.9 \pm 8.9$ \\
\hline Platycerium bifurcatum & Elkhorn fern & $17.8 \pm 3.4$ & $58.2 \pm 11.3$ \\
\hline Ardisia pusilla 'Variegata' & Variegated small coralberry & $11.9 \pm 3.9$ & $58.4 \pm 19.2$ \\
\hline Aphelandra squarrosa & Zebra plant & $17.9 \pm 2.4$ & $58.6 \pm 8.1$ \\
\hline Rhododendron simsii & Azalea & $18.4 \pm 0.9$ & $60.3 \pm 2.9$ \\
\hline Sansevieria trifasciata 'Superba' & Snake plant & $18.5 \pm 1.4$ & $60.4 \pm 10.3$ \\
\hline Peperomia quadrangularis 'Beetle' & Beetle peperomia & $19.0 \pm 4.8$ & $62.1 \pm 16.0$ \\
\hline Aglaonema 'Silver King' & Chinese evergreen 'Silver King' & $12.8 \pm 2.8$ & $62.7 \pm 14.1$ \\
\hline
\end{tabular}


Table 1. Continued.

\begin{tabular}{|c|c|c|c|}
\hline Botanical name & Common name & $\begin{array}{l}\text { Vmax } \\
(E T R)\end{array}$ & $\begin{array}{c}\mathrm{Km} \\
\left(\mu \mathrm{mol} \cdot \mathrm{m}^{-2} \cdot \mathrm{s}^{-1}\right)\end{array}$ \\
\hline Pentas lanceolata & Egyptian star cluster & $20.2 \pm 4.1$ & $66.3 \pm 13.5$ \\
\hline Cupressus macrocarpa 'Goldcrest' & Lemon cypress & $20.4 \pm 4.0$ & $67.2 \pm 13.3$ \\
\hline Schefflera actinophylla (Endl.) Harms & Umbrella tree & $21.8 \pm 1.6$ & $71.8 \pm 5.4$ \\
\hline Dischidia nummularia $\mathrm{R} . \mathrm{Br}$ & String of nickels & $22.3 \pm 2.4$ & $73.8 \pm 7.9$ \\
\hline Epipremnum aureum & Pothos & $22.5 \pm 4.9$ & $73.9 \pm 16.4$ \\
\hline Ardisia japonica 'Variegata' & Variegated small coralberry & $22.6 \pm 2.0$ & $74.2 \pm 6.6$ \\
\hline Fatsia japonica & Paperplant & $22.8 \pm 4.8$ & $74.9 \pm 15.8$ \\
\hline Ardisia japonica (Thunb.) Blume & Japanese ardisia & $23.1 \pm 2.2$ & $76.2 \pm 7.1$ \\
\hline Ficus benjamina 'Variegata' & Variegated benjamin fig & $20.1 \pm 2.1$ & $77.4 \pm 14.4$ \\
\hline Podocarpus nagi & Nagi podocarpus & $24.7 \pm 4.3$ & $81.2 \pm 14.3$ \\
\hline Tillandsia cyanea & Pink quill & $24.9 \pm 3.7$ & $82.0 \pm 12.2$ \\
\hline Hedera helix 'Variegata' & Variegated English ivy & $25.0 \pm 4.5$ & $82.4 \pm 14.9$ \\
\hline Hedera helix ‘Harley Quinn’ & Harley Quinn English ivy & $21.0 \pm 4.8$ & $85.3 \pm 32.1$ \\
\hline Hedera helix 'Dream' & Dream English ivy & $26.0 \pm 4.4$ & $85.6 \pm 14.7$ \\
\hline Pittosporum tobira & Japanese pittosporum & $25.9 \pm 0.8$ & $85.7 \pm 2.6$ \\
\hline Rhapis humilis Blume & Slender lady palm & $26.1 \pm 4.2$ & $86.2 \pm 13.9$ \\
\hline Euonymus japonicus 'Ovatus Aureus' & Japanese spindle & $26.4 \pm 1.1$ & $87.3 \pm 3.7$ \\
\hline Euphorbia pulcherrima & Poinsettia & $26.6 \pm 0.2$ & $87.6 \pm 0.8$ \\
\hline Ficus retusa 'Panda' & Ficus panda & $27.6 \pm 4.5$ & $90.9 \pm 15.1$ \\
\hline Nematanthus gregarius & Goldfish plant & $27.9 \pm 9.6$ & $91.6 \pm 31.8$ \\
\hline Heteropanax fragrans & Happy tree & $18.7 \pm 3.5$ & $92.2 \pm 17.6$ \\
\hline Nandina domestica Thunb. & Heavenly bamboo & $28.7 \pm 6.8$ & $94.6 \pm 22.5$ \\
\hline Serissa japonica (Thunb.) Thunb. & Snow rose & $29.3 \pm 5.1$ & $96.6 \pm 17.0$ \\
\hline Cyclamen persicum & Persian cyclamen & $29.9 \pm 3.6$ & $99.1 \pm 12.1$ \\
\hline Kalanchoe sp. & Kalanchoe & $30.6 \pm 4.7$ & $101.1 \pm 15.6$ \\
\hline Caladium sp. & Elephant ear & $31.0 \pm 3.3$ & $102.4 \pm 11.0$ \\
\hline Acalypha chamaedrifolia & Red cat's tail & $31.3 \pm 5.0$ & $103.3 \pm 16.6$ \\
\hline Ficus banghalensis & Bengal fig & $31.8 \pm 5.1$ & $105.2 \pm 17.1$ \\
\hline Trachelospermum asiaticum Nakai & Asiatic jasmine & $32.0 \pm 5.9$ & $105.8 \pm 19.7$ \\
\hline Pteris multifida Poir. & Spider brake fern & $22.0 \pm 4.8$ & $108.5 \pm 23.8$ \\
\hline Clusia rosea Jacq. & Balsam apple & $34.8 \pm 4.8$ & $115.0 \pm 15.9$ \\
\hline Eucalyptus globulus & Eucalyptus & $35.9 \pm 7.0$ & $118.8 \pm 23.4$ \\
\hline Hedera helix & Ivy & $24.0 \pm 0.7$ & $119.0 \pm 3.6$ \\
\hline Plectranthus tomentosa & Vick's plant & $37.3 \pm 6.5$ & $123.3 \pm 21.6$ \\
\hline Crossandra infundibuliformis (L.) Nees & Firecracker flower & $37.9 \pm 4.7$ & $125.2 \pm 15.6$ \\
\hline Polyscias scutellaria & Shield aralia & $38.7 \pm 6.4$ & $128.0 \pm 21.4$ \\
\hline Podocarpus macrophyllus (Thunb.) D. Don & Buddhist pine & $41.6 \pm 6.2$ & $137.9 \pm 20.9$ \\
\hline Pelargonium 'Vancouver Centennial' & Stellar geranium & $47.0 \pm 1.9$ & $155.6 \pm 6.4$ \\
\hline Fittonia verschaffeltii var. argyroneura 'Compacta' & Mosaic plant & $47.3 \pm 3.2$ & $156.8 \pm 10.5$ \\
\hline Murraya paniculata & Orange jasmine & $50.3 \pm 2.9$ & $166.6 \pm 9.7$ \\
\hline Stephanotis floribunda & Madagascar jasmine & $50.3 \pm 2.9$ & $167.0 \pm 9.7$ \\
\hline Mandevilla sanderi (Hemsl.) Woodson & Brazilian jasmine & $50.5 \pm 6.8$ & $167.2 \pm 22.5$ \\
\hline Adenium obesum & Desert rose & $53.0 \pm 3.2$ & $175.6 \pm 10.8$ \\
\hline Ceropegia woodii & Rosary vine & $60.2 \pm 8.9$ & $199.5 \pm 29.7$ \\
\hline Muehlenbeckia complexa Meisn. & Maidenhair vine & $68.7 \pm 4.3$ & $227.8 \pm 14.3$ \\
\hline Zamia pumila & Coontie palm & $53.0 \pm 3.2$ & $262.6 \pm 15.9$ \\
\hline Polyscias fruticosa & Ming aralia & $87.4 \pm 8.4$ & $290.2 \pm 28.1$ \\
\hline
\end{tabular}




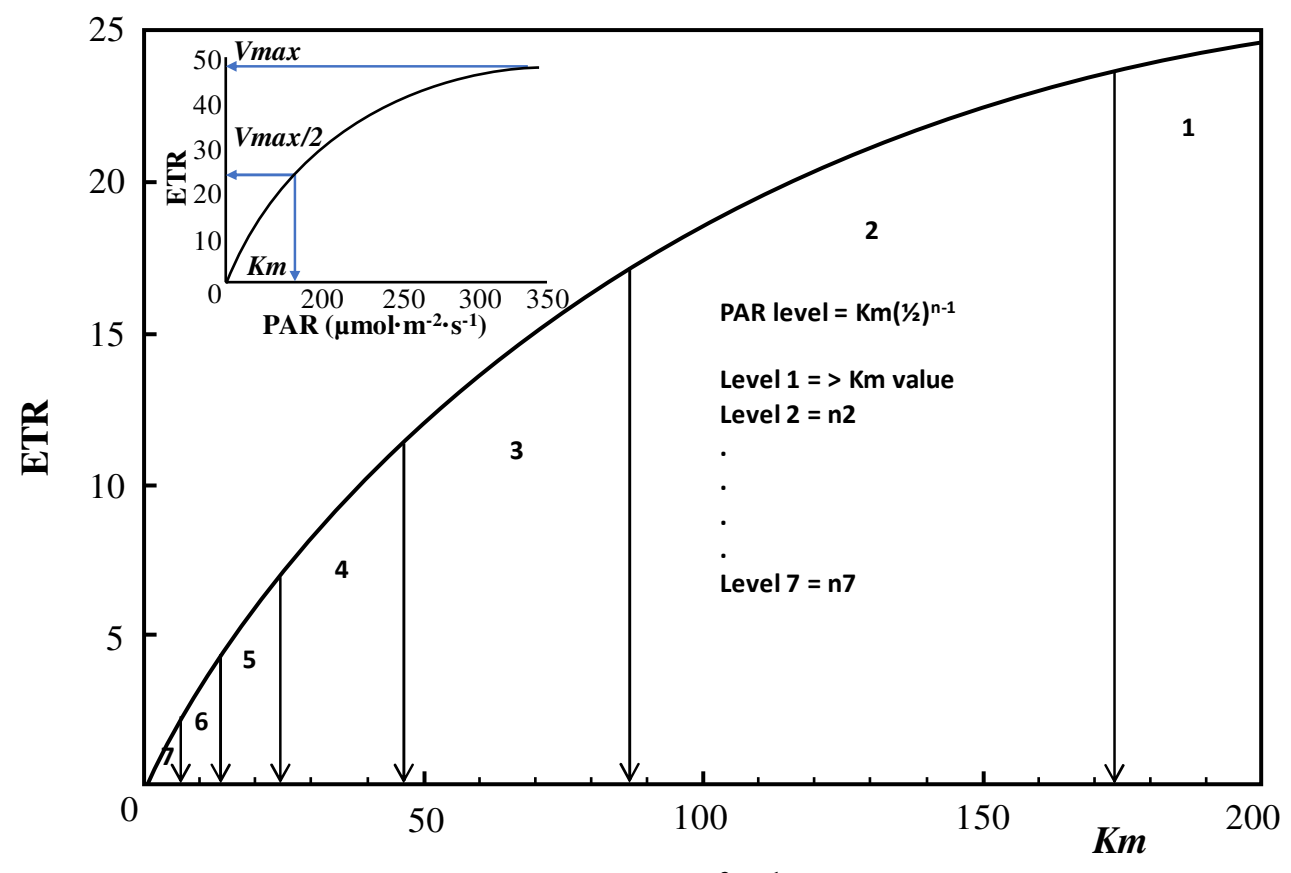

PAR $\left(\mu \mathrm{mol} \cdot \mathbf{m}^{-2} \cdot \mathbf{s}^{-1}\right)$

Figure 4. Formation of seven levels of light intensity according to the formula: $\mathrm{Km}\left(\frac{1}{2}\right)^{\mathrm{n}-1}$. Inset is $\mathrm{Vmax} / 2$ and $\mathrm{Km}$ in relation to the Vmax.

Table 2. Upper limit of light intensity per level of the 93 indoor plant species

\begin{tabular}{|c|c|c|c|c|c|c|c|}
\hline \multirow{2}{*}{ Common Name } & \multicolumn{7}{|c|}{ Upper limit of light intensity $\left(\mu \mathrm{mol} \cdot \mathrm{m}^{-2} \cdot \mathrm{s}^{-1}\right)$ per level } \\
\hline & 1 & 2 & 3 & 4 & 5 & 6 & 7 \\
\hline Belgian evergreen & $>20.9$ & 20.9 & 10.5 & 5.2 & 2.6 & 1.3 & 0.7 \\
\hline Tufted airplant & $>21.8$ & 21.8 & 10.9 & 5.4 & 2.7 & 1.4 & 0.7 \\
\hline Belgian evergreen 'Victoria' & $>25.2$ & 25.2 & 12.6 & 6.3 & 3.1 & 1.6 & 0.8 \\
\hline Mass cane & $>26.4$ & 26.4 & 13.2 & 6.6 & 3.3 & 1.7 & 0.8 \\
\hline Asparagus fern & $>28.3$ & 28.3 & 14.2 & 7.1 & 3.5 & 1.8 & 0.9 \\
\hline Painted-leaf begonia & $>28.4$ & 28.4 & 14.2 & 7.1 & 3.5 & 1.8 & 0.9 \\
\hline Neanthe bella palm & $>31.1$ & 31.1 & 15.5 & 7.8 & 3.9 & 1.9 & 1.0 \\
\hline Green congo philodendron & $>31.5$ & 31.5 & 15.7 & 7.9 & 3.9 & 2.0 & 1.0 \\
\hline Watermelon peperomia & $>31.6$ & 31.6 & 15.8 & 7.9 & 3.9 & 2.0 & 1.0 \\
\hline African violet & $>32.1$ & 32.1 & 16.0 & 8.0 & 4.0 & 2.0 & 1.0 \\
\hline Red aglaonema & $>32.1$ & 32.1 & 16.1 & 8.0 & 4.0 & 2.0 & 1.0 \\
\hline Butterfly plant & $>32.2$ & 32.2 & 16.1 & 8.0 & 4.0 & 2.0 & 1.0 \\
\hline Thick-stemmed wood fern & $>32.5$ & 32.5 & 16.2 & 8.1 & 4.1 & 2.0 & 1.0 \\
\hline Isabella peperomia & $>34.2$ & 34.2 & 17.1 & 8.6 & 4.3 & 2.1 & 1.1 \\
\hline Variegated croton & $>34.6$ & 34.6 & 17.3 & 8.7 & 4.3 & 2.2 & 1.1 \\
\hline Eastern teaberry & $>35.4$ & 35.4 & 17.7 & 8.9 & 4.4 & 2.2 & 1.1 \\
\hline Red star nerve plant & $>36.4$ & 36.4 & 18.2 & 9.1 & 4.6 & 2.3 & 1.1 \\
\hline Happy tree & $>36.6$ & 36.6 & 18.3 & 9.2 & 4.6 & 2.3 & 1.1 \\
\hline Prayer plant & $>38.4$ & 38.4 & 19.2 & 9.6 & 4.8 & 2.4 & 1.2 \\
\hline Good luck tree & $>38.9$ & 38.9 & 19.4 & 9.7 & 4.9 & 2.4 & 1.2 \\
\hline Variegated spikemoss & $>39.5$ & 39.5 & 19.8 & 9.9 & 4.9 & 2.5 & 1.2 \\
\hline
\end{tabular}


Table 2. Continued.

\begin{tabular}{|c|c|c|c|c|c|c|c|}
\hline \multirow{2}{*}{ Common Name } & \multicolumn{7}{|c|}{ Upper limit of light intensity $\left(\mu \mathrm{mol} \cdot \mathrm{m}^{-2} \cdot \mathrm{s}^{-1}\right)$ per level } \\
\hline & 1 & 2 & 3 & 4 & 5 & 6 & 7 \\
\hline Coral bead plant & $>39.8$ & 39.8 & 19.9 & 10.0 & 5.0 & 2.5 & 1.2 \\
\hline Broadleaf lady palm & $>43.0$ & 43.0 & 32.3 & 21.5 & 16.1 & 10.8 & 5.4 \\
\hline Rubber plant & $>43.8$ & 43.8 & 21.9 & 11.0 & 5.5 & 2.7 & 1.4 \\
\hline Wax begonia & $>44.3$ & 44.3 & 22.1 & 11.1 & 5.5 & 2.8 & 1.4 \\
\hline Mammy croton & $>44.4$ & 44.4 & 22.2 & 11.1 & 5.6 & 2.8 & 1.4 \\
\hline Bird's-nest fern & $>45.9$ & 45.9 & 22.9 & 11.5 & 5.7 & 2.9 & 1.4 \\
\hline Asiatic jasmine & $>46.0$ & 46.0 & 23.0 & 11.5 & 5.7 & 2.9 & 1.4 \\
\hline Variegated creeping fig & $>47.0$ & 47.0 & 23.5 & 11.7 & 5.9 & 2.9 & 1.5 \\
\hline Fiddle-leaf fig & $>47.6$ & 47.6 & 23.8 & 11.9 & 6.0 & 3.0 & 1.5 \\
\hline Small coralberry & $>48.1$ & 48.1 & 24.0 & 12.0 & 6.0 & 3.0 & 1.5 \\
\hline White star nerve plant & $>49.0$ & 49.0 & 24.5 & 12.3 & 6.1 & 3.1 & 1.5 \\
\hline Red gold Chinese evergreen & $>52.8$ & 52.8 & 26.4 & 13.2 & 6.6 & 3.3 & 1.6 \\
\hline Christmas berry & $>52.9$ & 52.9 & 26.5 & 13.2 & 6.6 & 3.3 & 1.7 \\
\hline Parasol plant & $>55.3$ & 55.3 & 27.7 & 13.8 & 6.9 & 3.5 & 1.7 \\
\hline Lucky bamboo & $>55.6$ & 55.6 & 27.8 & 13.9 & 6.9 & 3.5 & 1.7 \\
\hline Coffee tree & $>57.3$ & 57.3 & 28.7 & 14.3 & 7.2 & 3.6 & 1.8 \\
\hline Parallel peperomia & $>57.4$ & 57.4 & 28.7 & 14.3 & 7.2 & 3.6 & 1.8 \\
\hline Silver sprinkles & $>57.9$ & 57.9 & 29.0 & 14.5 & 7.2 & 3.6 & 1.8 \\
\hline Elkhorn fern & $>58.2$ & 58.2 & 29.1 & 14.5 & 7.3 & 3.6 & 1.8 \\
\hline Variegated small coralberry & $>58.4$ & 58.4 & 29.2 & 14.6 & 7.3 & 3.6 & 1.8 \\
\hline Zebra plant & $>58.6$ & 58.6 & 29.3 & 14.6 & 7.3 & 3.7 & 1.8 \\
\hline Azalea & $>60.3$ & 60.3 & 30.1 & 15.1 & 7.5 & 3.8 & 1.9 \\
\hline Snake plant & $>60.4$ & 60.4 & 30.2 & 15.1 & 7.5 & 3.8 & 1.9 \\
\hline Beetle peperomia & $>62.1$ & 62.1 & 31.1 & 15.5 & 7.8 & 3.9 & 1.9 \\
\hline Chinese evergreen 'Silver King' & $>62.7$ & 62.7 & 31.3 & 15.7 & 7.8 & 3.9 & 2.0 \\
\hline Egyptian star cluster & $>66.3$ & 66.3 & 33.2 & 16.6 & 8.3 & 4.1 & 2.1 \\
\hline Lemon cypress & $>67.2$ & 67.2 & 33.6 & 16.8 & 8.4 & 4.2 & 2.1 \\
\hline Umbrella tree & $>71.8$ & 71.8 & 35.9 & 17.9 & 9.0 & 4.5 & 2.2 \\
\hline String of nickels & $>73.8$ & 73.8 & 36.9 & 18.4 & 9.2 & 4.6 & 2.3 \\
\hline Pothos & $>73.9$ & 73.9 & 37.0 & 18.5 & 9.2 & 4.6 & 2.3 \\
\hline Variegated small coralberry & $>74.2$ & 74.2 & 37.1 & 18.6 & 9.3 & 4.6 & 2.3 \\
\hline Paperplant & $>74.9$ & 74.9 & 37.4 & 18.7 & 9.4 & 4.7 & 2.3 \\
\hline Japanese ardisia & $>76.2$ & 76.2 & 38.1 & 19.1 & 9.5 & 4.8 & 2.4 \\
\hline Variegated benjamin fig & $>77.4$ & 77.4 & 38.7 & 19.3 & 9.7 & 4.8 & 2.4 \\
\hline Nagi podocarpus & $>81.2$ & 81.2 & 40.6 & 20.3 & 10.2 & 5.1 & 2.5 \\
\hline Pink quill & $>82.0$ & 82.0 & 41.0 & 20.5 & 10.2 & 5.1 & 2.6 \\
\hline Variegated English ivy & $>82.4$ & 82.4 & 41.2 & 20.6 & 10.3 & 5.1 & 2.6 \\
\hline Harley Quinn English ivy & $>85.3$ & 85.3 & 42.7 & 21.3 & 10.7 & 5.3 & 2.7 \\
\hline Dream English ivy & $>85.6$ & 85.6 & 42.8 & 21.4 & 10.7 & 5.4 & 2.7 \\
\hline Japanese pittosporum & $>85.7$ & 85.7 & 42.8 & 21.4 & 10.7 & 5.4 & 2.7 \\
\hline Slender lady palm & $>86.2$ & 86.2 & 43.1 & 21.5 & 10.8 & 5.4 & 2.7 \\
\hline Japanese spindle & $>87.3$ & 87.3 & 43.7 & 21.8 & 10.9 & 5.5 & 2.7 \\
\hline Poinsettia & $>87.6$ & 87.6 & 43.8 & 21.9 & 10.9 & 5.5 & 2.7 \\
\hline Ficus panda & $>90.9$ & 90.9 & 45.4 & 22.7 & 11.4 & 5.7 & 2.8 \\
\hline Goldfish plant & $>91.6$ & 91.6 & 45.8 & 22.9 & 11.5 & 5.7 & 2.9 \\
\hline
\end{tabular}


Table 2. Continued.

\begin{tabular}{|c|c|c|c|c|c|c|c|}
\hline \multirow{2}{*}{ Common Name } & \multicolumn{7}{|c|}{ Upper limit of light intensity $\left(\mu \mathrm{mol} \cdot \mathrm{m}^{-2} \cdot \mathrm{s}^{-1}\right)$ per level } \\
\hline & 1 & 2 & 3 & 4 & 5 & 6 & 7 \\
\hline Happy tree & $>92.2$ & 92.2 & 46.1 & 23.0 & 11.5 & 5.8 & 2.9 \\
\hline Heavenly bamboo & $>94.6$ & 94.6 & 47.3 & 23.7 & 11.8 & 5.9 & 3.0 \\
\hline Snow rose & $>96.6$ & 96.6 & 48.3 & 24.2 & 12.1 & 6.0 & 3.0 \\
\hline Persian cyclamen & $>99.1$ & 99.1 & 49.5 & 24.8 & 12.4 & 6.2 & 3.1 \\
\hline Kalanchoe & $>101.1$ & 101.1 & 50.6 & 25.3 & 12.6 & 6.3 & 3.2 \\
\hline Elephant ear & $>102.4$ & 102.4 & 51.2 & 25.6 & 12.8 & 6.4 & 3.2 \\
\hline Red cat's tail & $>103.3$ & 103.3 & 51.7 & 25.8 & 12.9 & 6.5 & 3.2 \\
\hline Bengal fig & $>105.2$ & 105.2 & 52.6 & 26.3 & 13.1 & 6.6 & 3.3 \\
\hline Asiatic jasmine & $>105.8$ & 105.8 & 52.9 & 26.4 & 13.2 & 6.6 & 3.3 \\
\hline Spider brake fern & $>108.5$ & 108.5 & 54.3 & 27.1 & 13.6 & 6.8 & 3.4 \\
\hline Balsam apple & $>115.0$ & 115.0 & 57.5 & 28.8 & 14.4 & 7.2 & 3.6 \\
\hline Eucalyptus & $>118.8$ & 118.8 & 59.4 & 29.7 & 14.8 & 7.4 & 3.7 \\
\hline Ivy & $>119.0$ & 119.0 & 59.5 & 29.8 & 14.9 & 7.4 & 3.7 \\
\hline Vick's plant & $>123.3$ & 123.3 & 61.6 & 30.8 & 15.4 & 7.7 & 3.9 \\
\hline Firecracker flower & $>125.2$ & 125.2 & 62.6 & 31.3 & 15.6 & 7.8 & 3.9 \\
\hline Shield aralia & $>128.0$ & 128.0 & 64.0 & 32.0 & 16.0 & 8.0 & 4.0 \\
\hline Buddhist pine & $>137.9$ & 137.9 & 69.0 & 34.5 & 17.2 & 8.6 & 4.3 \\
\hline Stellar geranium & $>155.6$ & 155.6 & 77.8 & 38.9 & 19.5 & 9.7 & 4.9 \\
\hline Mosaic plant & $>156.8$ & 156.8 & 78.4 & 39.2 & 19.6 & 9.8 & 4.9 \\
\hline Orange jasmine & $>166.6$ & 166.6 & 83.3 & 41.6 & 20.8 & 10.4 & 5.2 \\
\hline Madagascar jasmine & $>167.0$ & 167.0 & 83.5 & 41.7 & 20.9 & 10.4 & 5.2 \\
\hline Brazilian jasmine & $>167.2$ & 167.2 & 83.6 & 41.8 & 20.9 & 10.4 & 5.2 \\
\hline Desert rose & $>175.6$ & 175.6 & 87.8 & 43.9 & 21.9 & 11.0 & 5.5 \\
\hline Rosary vine & $>199.5$ & 199.5 & 99.8 & 49.9 & 24.9 & 12.5 & 6.2 \\
\hline Maidenhair vine & $>227.8$ & 227.8 & 113.9 & 56.9 & 28.5 & 14.2 & 7.1 \\
\hline Coontie palm & $>262.6$ & 262.6 & 131.3 & 65.7 & 32.8 & 16.4 & 8.2 \\
\hline Ming aralia & $>290.2$ & 290.2 & 145.1 & 72.5 & 36.3 & 18.1 & 9.1 \\
\hline
\end{tabular}

Note. The values in each column are the upper limit of the range of light intensity in the specific level; e.g. for Belgian evergreens: Level 1: $>20.9$, Level 2: >10.5-20.9, Level 3: >5.2-10.5, Level 4: >2.6-5.2, Level 5:>1.3-2.6, Level 6:>0.7-1.3, and Level 7: 0-0.7 $\mu \mathrm{mol} \cdot \mathrm{m}^{-2} \cdot \mathrm{s}^{-1}$

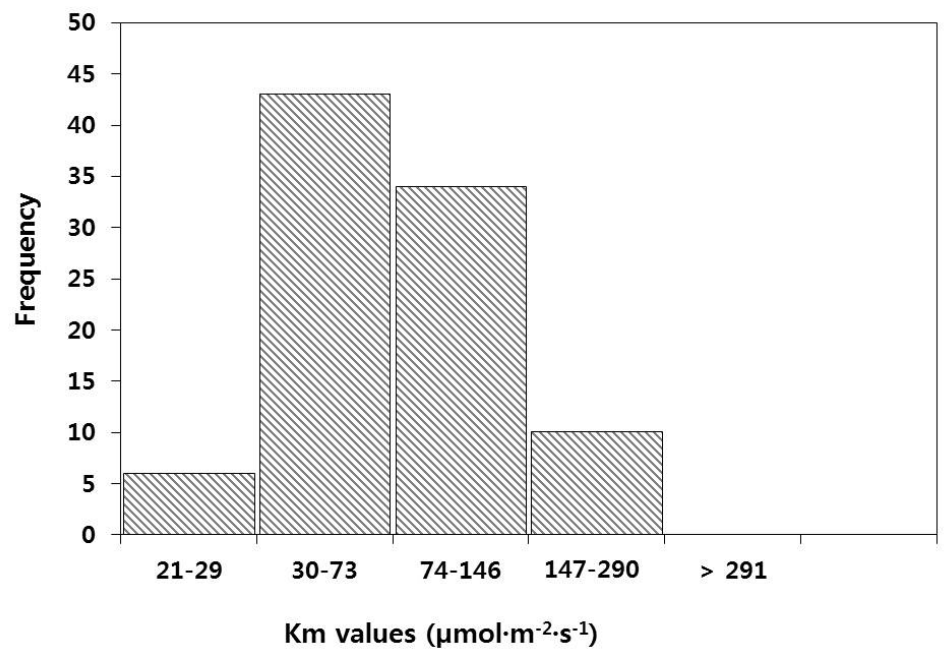

Figure 5. Histogram of $\mathrm{Km}$ values of different indoor plants. 
Majority of the studied indoor plants have $\mathrm{Km}$ values between 30 to $146 \mu \mathrm{mol} \cdot \mathrm{m}^{-2} \cdot \mathrm{s}^{-1} \mathrm{PAR}$ as shown in the histogram (Figure 5). In terms of light intensity requirement, this means that most of the plants require medium to high light intensity at about 1,554 to 6,660 lx. Fewer plant species require very low light and very high light intensities. The histogram was created using the different levels of light requirement of indoor plants (Pennisi, 2017)

Perhaps one of the difficulties in light intensity management of indoor plants is that different plants require different light intensities. Even within the same families, plant species may require varying illuminance as seen in Figure 6 . This is distinctly observed in families Acanthaceae, Apocynaceae, Araliaceae, and others.

There is a wide diversity of plants in a given light intensity. This is noticeable in light intensity ranges of 21-50 $\mu \mathrm{mol} \cdot \mathrm{m}^{-2} \cdot \mathrm{s}^{-1}, 56-90 \mu \mathrm{mol} \cdot \mathrm{m}^{-2} \cdot \mathrm{s}^{-1}$, and $91-125 \mu \mathrm{mol} \cdot \mathrm{m}^{-2} \cdot \mathrm{s}^{-1}$; with 19,19 , and 15 families represented, respectively (Figure 7). This shows that a homeowner can diversify his/her indoor plants with convenience if he/she knows the optimum light requirements of specific plants.

Despite the benefits gained from cultivation of indoor plants, homeowners may find it difficult or inconvenient to manage these especially for the working class. In terms of light quantity management, the inconvenience can be aggravated by changes in seasons, time of the days where natural light can enter the house, and others. Conventionally, the easiest way to adjust lighting is to move plants closer or farther away from the source of light. However, in smart homes, this becomes more convenient since adjustment of light intensity can be automated. This can be addressed by intelligent

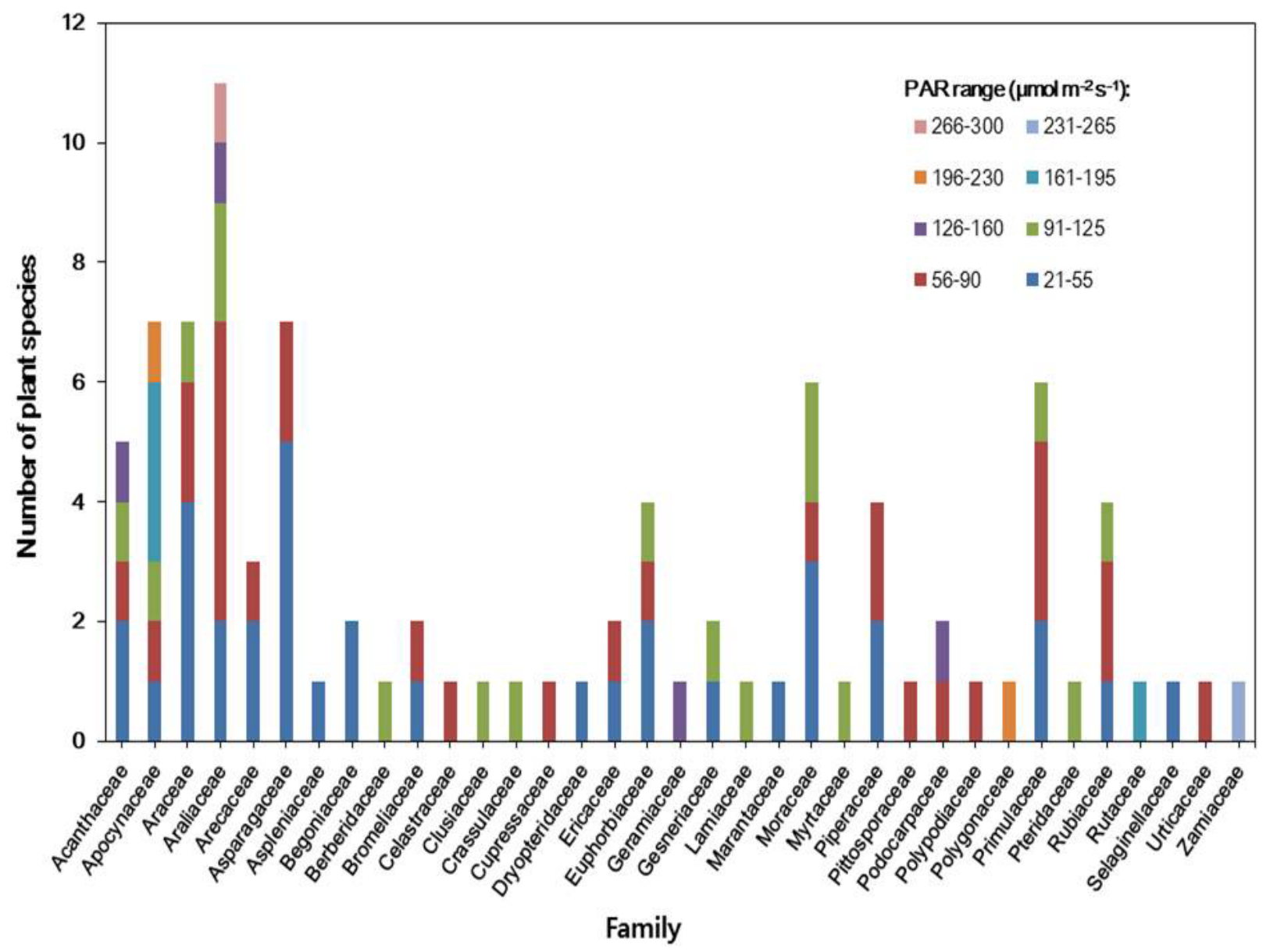

Figure 6. Number of plant species per family according to PAR range. 


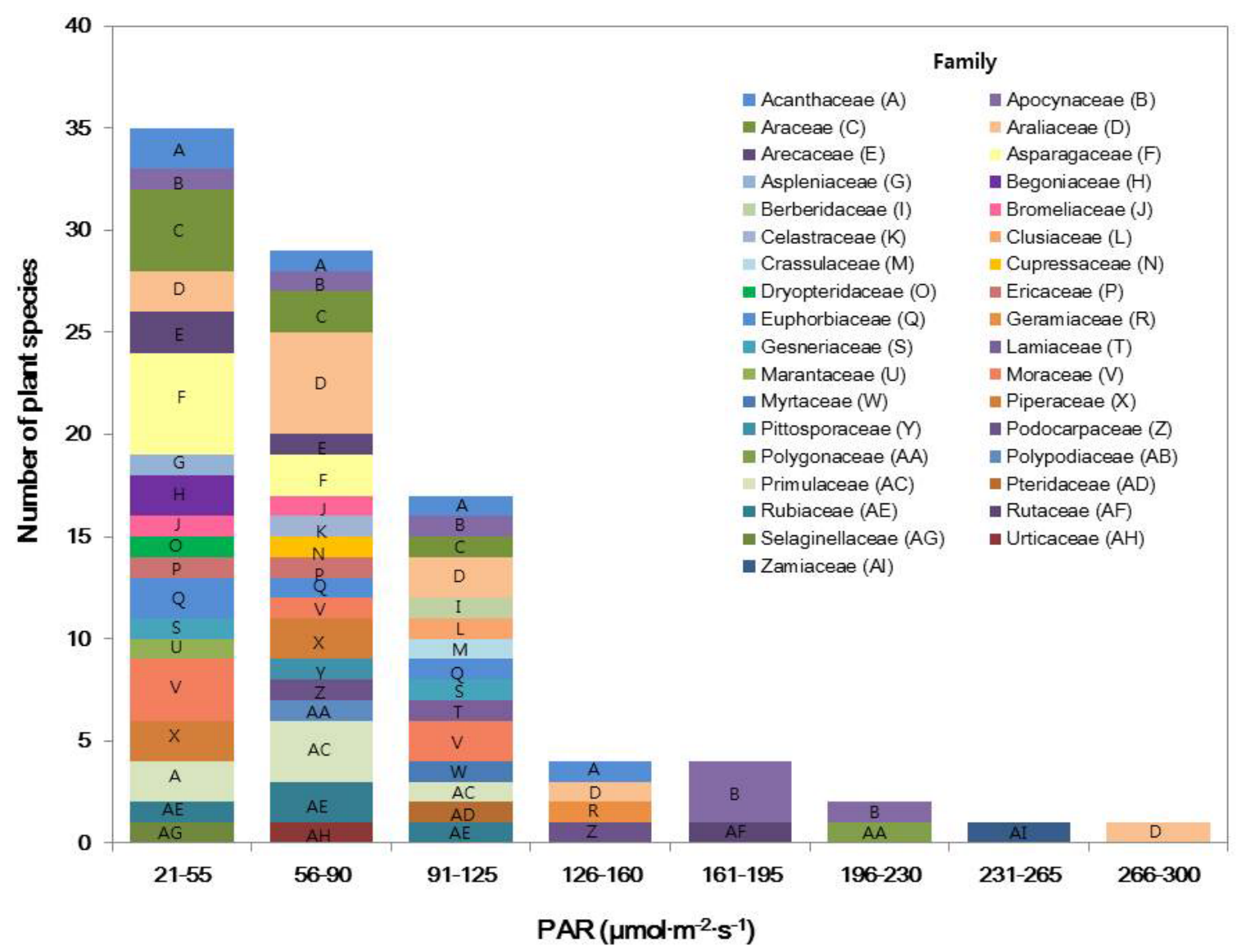

Figure 7. Distribution of indoor plant species grouped according to family per PAR range.

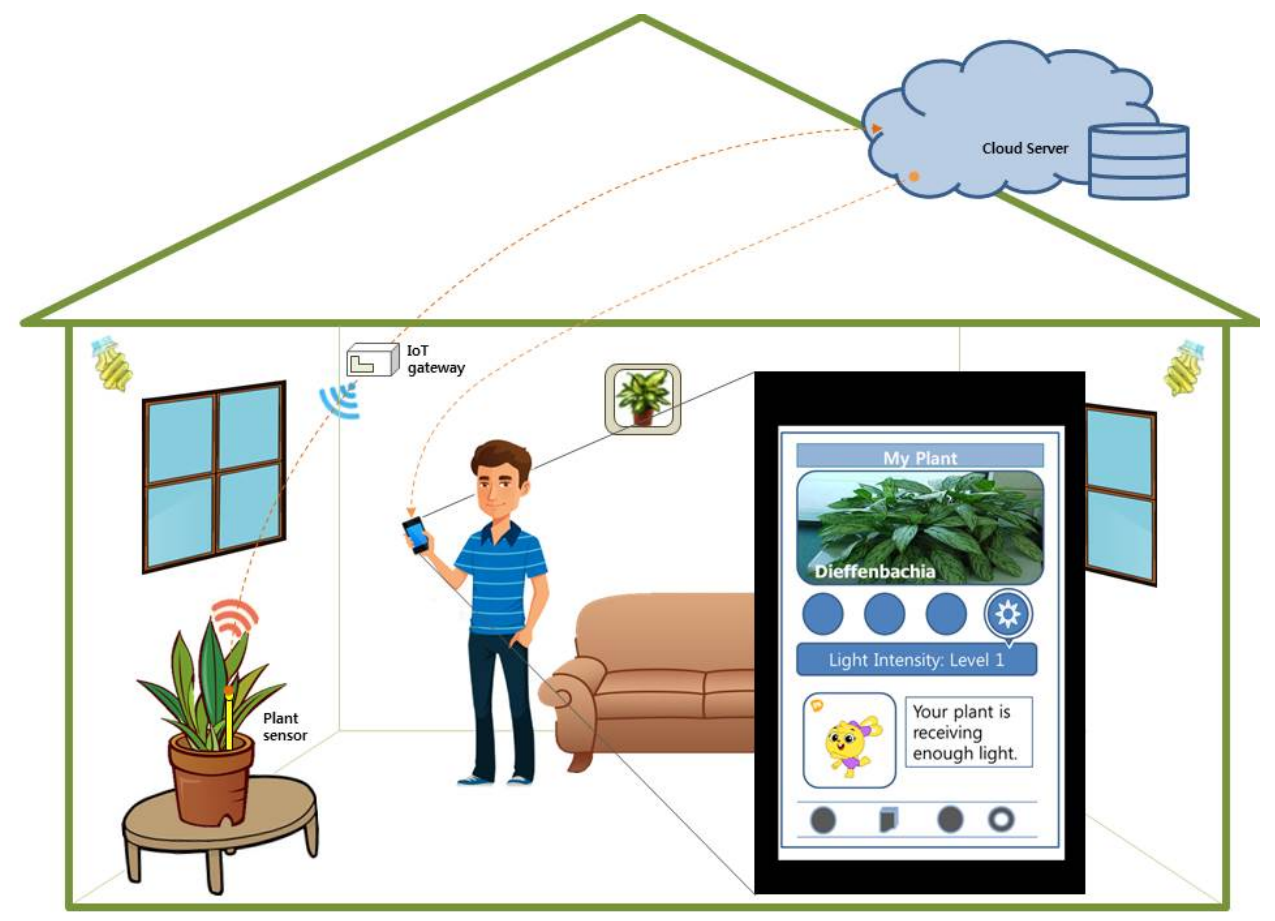

Figure 8. Concept of loT application for indoor plant cultivation in smart homes. 
monitoring by light or illuminance sensors which perceive the current environment and collect environmental data; controlled equipment such as smart switch for LED activate itself, adjusting the intensity of indoor light (Chen et al., 2017). The homeowners can monitor the status of their plants and their environment through smart gadgets such as smart phones (Figure 8). Yang et al. (2017) describes how botanical IoT technologies are being applied to agriculture and this can be applied to indoor environments like homes. Ray (2017) reviews several technologies involved in connecting plants to humans like sensing and controlling devices (e.g. Botanicalls, Parrot, etc.).

\section{Conclusion}

This study measured the photosynthetic response in terms of ETR of 93 indoor plants at different light intensity levels using chlorophyll fluorescence analysis. With the generated data we were able to create some ways to categorize the different indoor plants according to their optimum light intensity requirement, according to different light intensity range, distribution of plant species according to families, and diversity of plant families within a given PAR range. Specifically, seven levels of light intensity were formed from the generated data which may aide in the intelligence material needed in the IoT technology for smart homes that include greeneries or plant gardening in interior spaces. Although there are other factors influencing indoor plants like temperature and water requirements and effects of stress on these plants, our research is an initial step that can be valuable in the further advancement of indoor plant cultivation in smart homes.

\section{References}

Brilli, F., S. Fares, A. Ghirardo, P. de Visser, V. Calatayud, A. Munoz, I. Annesi-Maesano, F. Sebastiani, A. Alivermini, V. Varriale, and F. Menghini. 2018. Plants for sustainable improvement of indoor air quality. Trends Plant Sci. 23(6):507-512. DOI: 10.1016/j.tplants.2018.03.004

Channe, H., S. Kothari, and D. Kadam. 2015. Multidisciplinary model for smart agriculture using Internet-of-Things (IoT), sensors, Cloud-Computing, Mobile-Computing \& Big-Data Analysis Keywords. Int. J. Comput. Technol. Appl. 6(3):374-382.

Chen, M., J. Yang, X. Zhu, X. Wang, M. Liu, and J. Song. 2017. Smart Home 2.0: Innovative smart home system powered by botanical IoT and emotion detection. Mob. Netw. Appl. 22(6):1159-1169. DOI: 10.1007/s11036-017-0866-1

Irga, P.J., T.J. Pettit, and F.R. Torpy. 2018. The phytoremediation of indoor air pollution: a review on the technology development from the potted plant through to functional green wall biofilters. Rev. Environ. Sci. Biotechnol. 17(2):395-415. DOI: 10.1007/s11157-018-9465-2

Kim, K.J., M. Khalekuzzaman, J.N.Suh, H.J. Kim, C. Shagol, H.H. Kim, and H.J. Kim. 2018. Phytoremediation of volatile organic compounds by indoor plants: a review. Hortic. Environ. Biotechnol. 59(2):143-157. DOI: 10.1007/s13580-018-0032-0

Lee, M., J. Lee, B.J. Park, and Y. Miyazaki. 2015. Interaction with indoor plants may reduce psychological and physiological stress by suppressing autonomic nervous system activity in young adults: a randomized crossover study. J. Physiol. Anthropol. 34:21. DOI: 10.1186/s40101-015-0060-8

Pennisi, B.V. 2017. Growing Indoor Plants with Success. Athens, USA: University of Georgia Extension.

Pinyuh, B.G., E.B. Adams, L.A. Antonelli, and S.J. Collman. 2012. Indoor Plants. Kentucky Master Gardener Manual (pp. 195-208). Kentucky, USA: University of Kentucky.

Ray, P.P. 2017. Internet of things for smart agriculture: Technologies, practices and future direction. J. Ambient Intell. Smart Environ. 9(4):395-420. DOI: 10.3233/AIS-170440

Rohacek, K., J. Soukupova, and M. Bartak. 2008. Chlorophyll fluorescence: A wonderful tool to study plant physiology and plant stress. In: B. Schoefs (Ed.), Plant Cell Compartments-Selected Topics (pp. 41-104). Trivandrum, India: 
Research Signpost.

Shaughnessy, D. and A. Pertuit. 1999. Indoor plants- cleaning, fertilizing, containers and light requirements. South Carolina, USA: Clemson Cooperative Extension, Clemson University. Retrieved from https://hgic.clemson.edu/factsh eet/indoor-plants-cleaning-fertilizing-containers-light-requirements/

Shibata, S. and N. Suzuki. 2002. Effects of the foliage plant on task performance and mood. J. Environ. Psychol. 22(3):265 -272. DOI: 10.1006/jevp.2002.0232

Shibata, S. and N. Suzuki. 2004. Effects of an indoor plant on creative task performance and mood. Scand. J. Psychol. 45(5):373-381. DOI:10.1111/j.1467-9450.2004.00419.x

Yang, J., M. Liu, J. Lu, Y. Miao, M.A. Hossain, and M.F. Alhamid. 2017. Botanical internet of things: Toward smart indoor farming by connecting people, plant, data and clouds. Mob. Netw. Appl. 23(2):188-202. DOI: 10.1007/s11036-017-0930-x. 